
\title{
28 Research Suare \\ Integrated bioinformatics analysis and screening hub genes in papillary thyroid cancer
}

\section{Rong Fan}

Tianjin Xiqing hospital https://orcid.org/0000-0002-3388-0847

\section{Lijin Dong}

Logistics University of of People's Armed Police Force

\section{Ping Li}

southwest medical university

\section{Xiaoming Wang}

Tianjin institute of enviromental and operational medicine

Xuewei Chen ( $\boldsymbol{\sigma}$ chenxuewei11@sina.com )

https://orcid.org/0000-0001-6569-5630

\section{Research article}

Keywords: papillary thyroid cancer, hub gene, signaling pathway, survival analysis, expression profile

Posted Date: July 7th, 2020

DOI: https://doi.org/10.21203/rs.3.rs-35313/v1

License: (c) (1) This work is licensed under a Creative Commons Attribution 4.0 International License. Read Full License 


\section{Abstract \\ Background}

With the increasing incidence, papillary thyroid cancer (PTC) is receiving more and more attention, but the pathogenesis of which is still not completely elucidated. The purpose of this study was to explore key biomarkers and new therapeutic targets in PTC.

\section{Methods}

GEO2R and Venn online software were used for differential gene screening analysis. Hub genes were screened via STRING and Cytoscape, following Gene Ontology and KEGG enrichment analysis. Finally, survival analysis and expression validation were performed via UALCAN online software and immunohistochemistry.

\section{Results}

We screened 334 consistently differentially expressed genes (DEGs), composed of 136 upregulated genes and 198 downregulated genes. Gene ontology enrichment analysis suggested that DEGs mainly enriched in the cancer-related pathways and functions. PPI network visualization was performed to select 17 upregulated and 13 downregulated DEGs. Finally, the expression verification and overall survival analysis conducted in the Gene Expression Profiling Interactive Analysis Tool (GEPIA) and UALCAN showed that LPAR5, TFPI and ENTPD1 were related to the development of PTC and the prognosis of PTC patients, and the expression of LPAR5 was verified by tissue chip.

\section{Conclusions}

In summary, the hub genes and pathways identified in the present study not only provided new biomarkers for PTC, but also will be useful for elucidating the pathogenesis of PTC.

\section{Background}

For the past few years, more and more attention has been paid to thyroid diseases and the incidence of thyroid cancer has significantly increased [1]. The thyroid cancer is divided into five types according to histogenesis and morphology, including anaplastic, Hurthle cell, follicular, medullary and papillary thyroid cancer in which papillary thyroid cancer (PTC) accounts for $80 \%$ of the overall incidence [2]. With the widespread use of neck ultrasound and puncture, the number of mortality due to thyroid cancer is significantly reduced [3]. However, survival rate is affected by many factors, the prognosis of PTC is still very poor despite the use of multiple treatments such as thyroidectomy, radio-iodine treatment and chemistry-therapy [4]. Therefore, the early prevention and diagnosis of thyroid cancer is still an urgent 
problem for doctors and scientists, and it is very meaningful to explore potential key biomarkers and novel therapeutic targets in PTC for doctors and patients.

With the large-scale application of high-throughput screening technology, we have found many novel genes associated with disease initiation and progression and had a deep understanding of the molecular mechanisms of various tumor development [5-8]. In present study, we used the bioinformatics methods to mine the microarray data from GEO, analyzed the DEGs between PTC and normal tissues. Then, we built the protein-protein interaction (PPI) network, performed the analysis of functions enrichment and survival analysis, and identified 3 hub genes and discovered the biological processes and signaling pathways associated with PTC. In conclusion, the integrated analysis provided some new biomarkers for PTC, which could be valuable for the further research on the mechanism and the clinical application of diagnosis, prognosis and therapy of PTC.

\section{Methods}

\subsection{Acquisition of microarray data}

We obtained the high-throughput gene expression profiles of PTC and normal thyroid tissues from the GEO database. The independent datasets of GSE3678, GSE33630 and GSE53157 were selected and all of them were based on the GPL570 Platformsincluding 7 PTC tissues and 7 normal tissues, 49 PTC tissues and 45 normal tissues and 7 PTC tissues and 3 normal tissues, respectively.

\subsection{Screening of DEGs}

The DEGs between PTC tissues and normal tissues were screened via GEO2R tool. The cut-off criteria of $\square \log \mathrm{FC} \otimes \geq 1$ and adjust $P$-value $<0.05$ were considered statistically significant. Then, the DEGs in three datasets were screened out by Venn software online. The DEGs with $\square \log F C \unrhd \geq 1$ were considered as upregulated genes, while $\mathrm{DEGs}$ with $\square \log \mathrm{FC} \varangle<1$ were considered as down-regulated genes.

\subsection{Enrichment analysis via GO and KEGG pathway}

In order to characterize the functional roles of the DEGs, we used the Database for Annotation, Visualization, and Integrated Discovery (DAVID, version 6.8) [9] for GO enrichment analysis which included biological process (BP), cellular component (CC), molecular function (MF), and KEGG pathways analysis with a cut-off of $P$-value $<0.05$.

\subsection{Construction of PPI network and analysis of module}

The PPI network was built via the Search Tool for the Retrieval of Interacting Genes/Proteins (STRING) database to construct to uncover the relationships of DEGs based on a minimum required interaction score $=0.4$ [10]. PPI network was displayed and analyzed by Cytoscape (version 3.6.1) software [11]. In addition, the core modules of the PPI network were screened via the MCODE (node score cutoff $=0.2$, degree cutoff $=2$, $k$-score $=2$, and max. Depth $=100$ ). 


\subsection{Survival analysis and validation of the hub genes expression}

UALCAN was used for analyzing the relationship between key genes expression and survival of patients with PTC, which is a good resource for analyzing transcriptome data of cancers from The Cancer Genome Altas (TCGA) [12]. The Gene Expression Profiling Interactive Analysis tool (GEPIA) [13] was used for analyze RNA expression data on the basis of thousands of samples from TCGA and the GTEx projects. $P$-value $<0.05$ was considered statistically significant.

\subsection{Tissue samples and immunohistochemistry (IHC)}

Human papillary thyroid cancer tissue microarray sections (HThyP120CS02) was obtained from Shanghai Outdo Biotech Co. Ltd. (Shanghai, China). The tissue samples were procured from 62 patients with papillary thyroid cancer which consisted of 58 cancer tissues and 58 para-cancerous tissues as well as 3 normal thyroid tissues and 1 chronic lymphocytic thyroiditis. The two-step EnVision method was used to perform immune histochemical experiments, along with different primary antibodies against LPAR5 (1:50). The study was approved by the Ethics Committee of Shanghai Outdo Biotech Company (Shanghai, China). The slides were analyzed using the Image-Pro PLUS software program (Media Cybernetics, Inc. USA).

\subsection{Statistical analysis}

Statistical analysis was performed via SPSS 22.0 and GraphPad Prism 8.0 software. Results were presented as the mean \pm standard deviation. The statistically significant changes were indicated with asterisks and the $P$-values were calculated via a Student's t-test, wherein *, $* *$, and $* \star *$ represented $P<$ $0.05, P<0.01$ and $P<0.001$, respectively.

\section{Results}

Identification of DEGs in papillary thyroid cancers

In this study, we selected and downloaded three GEO datasets and extracted DEGs based on the cut-off criteria. A total of 63 papillary thyroid cancer and 55 normal thyroid tissues were obtained in this study. There were 636 DEGs in GSE3678, including 271 upregulated and 365 downregulated genes, 1241 DEGs in GSE33630, including 673 upregulated and 568 downregulated genes, and 884 DEGs in GSE53157, including 369 upregulated and 515 downregulated genes. By using Venn diagram software, we detected a total 334 commonly DEGs, which included 136 upregulated genes and 198 downregulated genes in the PTC tissues (Table 1 \& Fig. 1). 
Table 1

334 commonly differentially expressed genes were screened from three profile datasets, including 136 upregulated and 198 downregulated genes in PTC tissues compared to normal thyroid tissues.

\section{DEGs Genes symbol}

Upregulated

CDH3, KLHDC8A, KCNJ2, GOLT1A, TNIK, PDZK1IP1, BID, RXRG, MPZL2, GDF15, FRMD3, DOCK9, MET, PTPRE, FAM20A, IGSF3, ALOX15B, ALOX5, EPS8, NGEF, ITGA3, DUSP6, PRSS2, CD55, NOD1, CFI, DDB2, DPP4, NPC2, DUSP4, CORO2A, ATP11A, ST8SIA4, LGALS3, PDLIM4, PLAG1, SYT12, RASD2, NFE2L3, TTC39B, DUSP5, LPAR5, TUSC3, FAXC, MYEF2, PDE5A, TACSTD2, PRR15, CDC42EP3, SNX25, RYR1, SPINT1, LRRK2, LOC101927705///P4HA2, NRP2, PRSS23, SIPA1L2, CHI3L1, CITED1, C19orf33, NR2F1-AS1, SERPINA1, TGFA, PLXNC1, LIPH, GGCT, LONRF2, TIAM1, FN1, SYTL1, RUNX2, SNX22, C4orf48, SYTL5, SERINC2, KRT19, ZCCHC12, LAMB3, LMO3, TENM1, SHROOM4, EMILIN2, ENTPD1, GALE, SDC4, METTL7B, HEY2, ETV5, ABCC3, NRCAM, LOC101928269///LOC100506403///RUNX1, GABRB2, SLC35F2, TNRC6C-AS1, TMPRSS4, SLC34A2, BICD1, STK32A, MYH10, RAB27A, TBC1D2, LRP4, CLDN1, LOC729461///FAM230B///FAM230C, TGFBR1, GABBR2, CCND1, NHSL2, MEGF9, CDH6, GALNT7, SREBF1, PTP4A3, TMC6, TPD52L1, PROS1, CAMK2N1, QPCT, SCEL, MCTP2, ETV1, KCNN4, KDELR3, PCSK2, COL8A1, ZMAT3, CYP1B1, HMGA2, LOC101928195///LOC100996643///MTHFD1L, LOC100507165, PSD3, AGR2, DTX4, CTSC, ARMCX3, XPR1

downregulated

HBA2///HBA1, PKNOX2, MUM1L1, COL9A3, PBX4, IRS4, FGL2, MPPED2, DPT, TBC1D4, CITED2, ASXL3, MFAP4, FOXP2, FAM234B, DLG2, LAYN, TNS3, STXBP5L, SCARA5, TCEAL2, GPR83, RGS16, HGD, PAPSS2, GLT8D2, ID4, DGKI, EML1, GJB6, CDH16, AN05, GHR, GNAI1, SCUBE3, LIFR, GRAMD2, MAGI2, CSGALNACT1, LYVE1, LOC101930400///AKR1C2, TMPRSS3, SOD3, GDF10, TFCP2L1, OTOS, EML6, STARD13, BCL2, KIAA1324, CXCL12, IPCEF1, RAP1GAP, PLEKHG4B, 2-Mar, AVPR1A, WDR72, SPX, IRS1, TSPAN7, PID1, SMAD9, BTBD11, C4orf47, ELM01, NCAM1, DIO2, TPO, CASZ1, SORD, PQLC2L, SLC25A33, FCGBP, TBC1D8///RPL31, RYR2, PLA2R1, PKHD1L1, KCNIP4, GSTM3, ESRRG, CHCHD10, HBB, SERTM1, FHL1, RHOJ, SORBS2, MAFB, IP6K3, TFPI, C11 orf74, RPS6KA6, MT1G, TMEM171, PKIA, PEG3, CCL21, ST7-AS1, SCN3A, CAPSL, PPARGC1A, DEPTOR, FBLN7, ER01B, CRABP1, ZFPM2, RNF157-AS1, FHDC1, GNA14, MT1F, SHANK2, ANKS1B, BMP8A, COL23A1, TGFBR3, ADH1B, AKR1C1, ANK2, NUAK2///AKIP1, KIZ, VLDLR, LMOD1, PRKCQ, DI01, CCDC146, FGFR2, KIT, FABP4, ZDHHC11B///ZDHHC11, FAM167A, FAXDC2, ACACB, LOC440934, LOC646736, CYP7B1, RGS8, PGM5, MIR4683///FZD8, AADACP1, RNF150, AGPAT4, ZMAT4, TBX22, SLC16A2, TPPP, LOC101927137///KIAA1456, CTH, RASSF9, DDX25, TLE4, AKR1C3, SH3GL2, LOC101929480, DIRAS2, AGTR1, FMOD, ADAM22, LOC101060817///GCSH, DPY19L2, CLMN, SNCA, LRIG1, ANKRD18A, AIF1L, L3MBTL4, ADGRV1, RELN, CWH43, IGFBPL1, LOC100506558///MATN2, SLC26A7, WWOX, WASF3, UGT8, CUX2, SOX9-AS1, SLC4A4, TMEM178A, OGDHL, ADGRA3, DYNLRB2, TFF3, AOX1, GPM6A, CLCNKB, MDH1B, SAMD5, CFD, DPP6, TRIM58, INAFM2, FLRT1, MR0, TTC30A, EFEMP1, WFS1, SMOC2, ANKRD37

\section{GO and pathway enrichment analysis}

We analyzed the upregulated and downregulated genes by DAVID software and identified 125 significant enrichments terms including biological processes (BP, 79), molecular functions (MF, 22) and cellular components (CC, 24). For BP, DEGs were significantly enriched in cell adhesion, positive regulation of gene expression, BMP signaling pathway, transforming growth factor beta receptor signaling pathway, SMAD protein signal transduction, positive regulation of MAP kinase activity, ventricular septum morphogenesis, endothelial cell migration, melanocyte differentiation, and so on. For MF, DEGs were 
significantly enriched in calcium ion binding, protease binding, glycoprotein binding, serine-type peptidase activity, RNA polymerase $\triangle$ transcription coactivator activity, growth factor binding, MAP kinase tyrosine/serine/threonine phosphatase activity and semaphorin receptor activity. For CC, DEGs were remarkably enriched in plasma membrane, extracellular space, cell surface, proteinaceous extracellular matrix, neuronal cell body, apical plasma membrane, receptor complex, dendritic spine and costamere. The top $10 \mathrm{GO}$ terms were shown in Fig. 2. Furthermore, KEGG pathway analysis showed that the DEGs were enriched in 8 pathways including pathways in cancer, Rap1 signaling pathway, transcriptional misregulation in cancer, insulin resistance, small cell lung cancer, adipocytokine signaling pathway, complement and coagulation cascades and tyrosine metabolism (Fig. 2).

\section{Construction of PPI network and analysis of module}

By STRING and Cytoscape analysis, we drew the PPI network complex constructed by 241 nodes and 442 edges, including 101 upregulated and 137 downregulated genes. Then we made further analysis by applying Cytoscape MCODE plus and found 30 central nodes including 17 upregulated genes and 13 downregulated genes (Fig. 3, 4 \& Table 2).

Table 2

30 central genes were selected by STRING and Cytoscape software from PPI network including 17 upregulated and 13 downregulated genes.

\begin{tabular}{|ll|}
\hline DEGs & Gene symbol \\
\hline upregulated & $\begin{array}{l}\text { CCND1, FAM20A, FN1, PRSS23, LPAR5, SERPINA1, KRT19, GABBR2, RUNX2, } \\
\text { CHI3L1, CFI, QPCT, CD55, CYP1B1, RAB27A, ENTPD1, COL8A1 }\end{array}$ \\
\hline Downregulated & $\begin{array}{l}\text { GNAI1, NCAM1, CCL21, IRS1, WFS1, FGFR2, FGL2, AKR1C3, AKR1C1, TFF3, } \\
\text { COL23A1, TFPI, COL9A3 }\end{array}$ \\
\hline
\end{tabular}

\section{Selection of hub genes and Validation of the expression level}

We used GEPIA to identify 30 central genes survival data and found that the expression levels of 4 hub genes were significantly associated with survival of thyroid cancer patients which included ENTPD1, LPAR5, AKR1C3 and TFPI ( $P<0.05$, Fig. 5). Furthermore, GEPIA was applied to validate the expression level of 4 hub genes in PTC and normal tissue respectively. Results showed that the expression of all the four genes were remarkably different in PTC samples contrasted to normal samples. ENTPD1 and LPAR5 expression levels were significantly upregulated while the expression levels of AKR1C3 and TFPI were remarkably downregulated in PTC samples than normal samples (Fig. 6). Moreover, UALCAN was used to analyze the expression levels of the four hub genes. We found that the expression levels of ENTPD1, LPAR5 and TFPI were remarkably different in PTC compared to normal tissue except for AKR1C3 (Fig. 7). In order to verify the reliability of the prediction, we selected LPAR5 protein and analyzed its expression by using tissue microarray. The results indicated that LPAR5 expression appeared to be remarkably higher in papillary thyroid cancer tissues than in the adjacent tissues (Fig. 8).

\section{Discussion}


In this study, we analyzed gene expression profiles from three GEO datasets including GSE 3678, GSE 33630 and GSE 53157 by GEO2R and identified 136 upregulated and 198 downregulated genes, for a total of 334 DEGs. In particular, the data sets we selected are from the same data platform, and the purpose is to ensure data uniformity and reliability. 63 papillary thyroid cancer specimens and 55 normal thyroid specimens were enrolled in this research. Gene Ontology and KEGG pathway enrichment analysis suggested that these DEGs were remarkably enriched in some pathways including pathways in cancer, TGF $\beta$ receptor signaling pathway, growth factor binding and closely associated with BMP, SMAD, MAPK signal activity. Then, we constructed PPI network via STRING and Cytoscape software and screened 30 vital hub genes from the PPI network complex via MCODE module analysis. Furthermore, we found that 4 of 30 genes were closely associated with survival by GEPIA analysis. Expression validation from GEPIA and UALCAN showed that there was a significant difference in the expression of ENTPD1, LPAR5 and TFPI in PTC and normal tissue, which suggested that these genes might be critical in the tumorigenesis and development of PTC.

Previous research had indicated that the three genes screened by our study were involved in the initiation and development of tumors. Lysophosphatidic acid (LPA) is a biologically active mediator that affects cellular functions such as regulating cell proliferation, transcellular migration, differentiation, morphogenesis, and preventing apoptosis $[14,15]$. LPAR5 is a member of $\mathrm{G}$ protein-coupled transmembrane receptors which interact with LPA $[16,17]$. The cells treated by $12-0-$ tetradecanoylphorbol-13acetate (TPA) showed higher motile activity than control cells, while LPAR5 knockdown reversed the phenomenon [18]. Recent studies on PTC showed that LPAR5 was associated with progression and overall survival in thyroid cancer, which is consistent with our study [19-21]. Tissue factor pathway inhibitor (TFPI), is the endogenous inhibitor of tissue factor induced blood coagulation, and its expression had been demonstrated in smooth muscle cells, monocytes, plate lets and several breast cancer cell lines [22-24]. Wang et al. suggested that down-regulation of TFPI may result in the tumor cell growth and migration, the suppression of TFPI by hypoxia microenvironment might be one of the supervise mechanisms by which hypoxia promotes the angiogenesis process and tumor growth [25]. miR-500 inhibition could surpress the proliferation and invasion prostate cancer cell and tumorigenicity in vivo, while TFPI knockdown reversed the effects [26]. The level of TFPI in luminal-A breast cancer patients is significantly lower than healthy volunteers [27]. ZARYCHTA et al. claimed that TF seemed to be a tumor-promoting factor while TFPI exerted tumor suppressor properties [28]. Cihangir et al. found that a significant decrease in TFPI levels in hyperthyroidism patients [29]. ENTPD1, which encodes for the protein CD39, is very important to the production of immunsuppressive adenosine [30]. Eman et al. confirmed that the level of ENTPD1 on CD4 positive T helper cells in chronic lymphocytic leukemia (CLL) patients was significantly higher compared to the controls and ENTPD1 and CD4 were remarkably expressed in high risk CLL patients [31]. Cai et al. found that overexpression of ENTPD1 is a predictor of poor prognosis for $\mathrm{GC}$ patients after radical gastric cancer $(\mathrm{GC})$ resection [32]. The pathological research showed that the expression of ENTPD1 in squamous cell carcinoma of the head and neck (HNSCC) is positively correlated with tumor stage, which may predict a poor prognosis [33]. Interestingly, Bastid et al. found that ENTPD1 deletion promoted development of both induced and spontaneous autochthonous 
liver cancer in mice [34]. All these suggest that the immunotherapy treatment targeting CD39 may be promising. So far, there are few reports on the relationship between ENTPD1 and thyroid cancer. Thus, the specific functions of ENTPD1 in thyroid cancer were worth to be further explored. However, further experiments will be necessary to explore pathogenesis basis and molecular mechanism of these hub genes in PTC.

\section{Conclusions}

In summary, our integrated bioinformatics study presented 3 hub genes (LPAR5, TFPI and ENTPD1) and pathways associated with PTC, which may be a reliable and potential biomarker and provide new insights into the diagnosis, prognosis and target therapy for PTC.

\section{Abbreviations}

PTC: Papillary thyroid cancer; GEO:Gene Expression Omnibus; DEGs:Different expressed genes; DAVID:Database for Annotation, Visualization and Integrated Discovery; GO:Gene ontology; KEGG:Kyoto Encyclopedia of Gene and Genome; BP:Biological process; CC:Cellular component; MF:Molecular function; STRING:Search Tool for the Retrieval of Interacting Genes/Proteins; MCODE:Molecular Complex Detection; GEPIA:Gene Expression Profiling Interactive Analysis; TCGA:The Cancer Genome Altas; AKR1C3:Aldo-Keto Reductase Family 1 Member C3; LPAR5:Lysophosphatidic Acid Receptor 5; TFPI:Tissue Factor Pathway Inhibitor; ENTPD1:Ectonucleoside Triphosphate Diphosphohydrolase 1; MAPK:Mitogen-Activated Protein Kinase; BMP:Bone Morphogenetic Protein; TPA:12-0tetradecanoylphorbol-13acetate; CLL:chronic lymphocytic leukemia; GC:gastric cancer; HNSCC:squamous cell carcinoma of the head and neck.

\section{Declarations}

\section{Ethics approval and consent to participate}

All work were conducted in accordance with the Declaration of Helsinki (1964). The experiment was conducted with the human subjects' understanding and consent and was approved by the Ethics Committee of Tianjin Xiqing hospital. This study has obtained written informed consent from the participants

\section{Consent for publication}

The work described has not been published before. It is not under consideration for publication elsewhere. Its publication has been approved by all co-authors. Its publication has been approved by the responsible authorities at the institution where the work is carried out.

\section{Availability of data and material}


The microarray data used to support the findings of this study have been deposited in the Gene Expression Omnibus (GEO) repository (accession numbers: GSE3678, GSE33630 and GSE53157).

\section{Competing interests}

The authors have declared that no conflict of interest exists.

\section{Funding}

The sample collection work was completed in Tianjin Xiqing hospital, and supported by the National Natural Science Foundation of China (No. 81201757 and 81902715). The equipment required for bioinformatics analysis was supported by the Basic research projects of Logistic University of Chinese People's Armed Police Force, No.WHJ201702. The immunohistochemistry experiment was completed and supported by Tianjin Institute of Environmental and Operational Medicinesupported (AWS16J004). The content is solely the responsibility of the authors and does not necessarily represent the official views of the National Institutes of Health. These funding bodies support the data collection and analysis.

\section{Authors' Contributions}

R.F conceived the idea to this paper; L.D and P.L collected and analyzed the data; X.W drafted the paper; $X . C$ revised the final paper.

\section{Acknowledgements}

The visualization of GO analysis, including BP, MF and CC of DEGs, was performed via the online software ImageGP (http://www.ehbio.com/ImageGP/index.php/Home/Index/index).

\section{References}

1. Siegel RL, Miller KD, Jemal A. Cancer Statistics. 2017. CA: a cancer journal for clinicians. 2017;67(1):7-30.

2. Tuttle RM, Ball DW, Byrd D, Dilawari RA, Doherty GM, Duh QY, et al. Thyroid carcinoma Journal of the National Comprehensive Cancer Network: JNCCN. 2010;8(11):1228-74.

3. La Vecchia C, Malvezzi M, Bosetti C, Garavello W, Bertuccio P, Levi F, et al. Thyroid cancer mortality and incidence: a global overview. International journal of cancer. 2015;136(9):2187-95.

4. Schlumberger M, Sherman SI. Approach to the patient with advanced differentiated thyroid cancer. European journal of endocrinology. 2012;166(1):5-11.

5. Vogelstein B, Papadopoulos N, Velculescu VE, Zhou S, Diaz LA Jr, Kinzler KW. Cancer genome landscapes. Science. 2013;339(6127):1546-58. 
6. Zhao P, Hu W, Wang H, Yu S, Li C, Bai J, et al. Identification of differentially expressed genes in pituitary adenomas by integrating analysis of microarray data. International journal of endocrinology. 2015;2015:164087.

7. Gao X, Wang X, Zhang S. Bioinformatics identification of crucial genes and pathways associated with hepatocellular carcinoma. Bioscience reports. 2018;38(6).

8. Sun $C$, Cheng $X$, Wang C. Gene expression profiles analysis identifies a novel two-gene signature to predict overall survival in diffuse large B-cell lymphoma. 2019;39(1).

9. Huang da W, Sherman BT, Lempicki RA. Systematic and integrative analysis of large gene lists using DAVID bioinformatics resources. Nature protocols. 2009;4(1):44-57.

10. Szklarczyk D, Morris JH, Cook H, Kuhn M, Wyder S, Simonovic M, et al. The STRING database in 2017: quality-controlled protein-protein association networks. made broadly accessible. 2017;45(D1):D362-d8.

11. Shannon P, Markiel A, Ozier O, Baliga NS, Wang JT, Ramage D, et al. Cytoscape: a software environment for integrated models of biomolecular interaction networks. Genome research. 2003;13(11):2498-504.

12. Chandrashekar DS, Bashel B, Balasubramanya SAH, Creighton CJ, Ponce-Rodriguez I, Chakravarthi B, et al. UALCAN: A Portal for Facilitating Tumor Subgroup Gene Expression and Survival Analyses. Neoplasia (New York). 2017;19(8):pp. 649-58.

13. Tang Z, Li C, Kang B, Gao G, Li C, Zhang Z. GEPIA: a web server for cancer and normal gene expression profiling and interactive analyses. Nucleic acids research. 2017;45(W1):W98-w102.

14. Furui $T$, LaPushin $R$, Mao $M$, Khan $H$, Watt $S R$, Watt MA, et al. Overexpression of edg-2/vzg- 1 induces apoptosis and anoikis in ovarian cancer cells in a lysophosphatidic acid-independent manner. Clinical cancer research: an official journal of the American Association for Cancer Research. 1999;5(12):4308-18.

15. Contos JJ, Ishii I, Fukushima N, Kingsbury MA, Ye X, Kawamura S, et al. Characterization of Ipa(2) (Edg4) and Ipa(1)/lpa(2) (Edg2/Edg4) lysophosphatidic acid receptor knockout mice: signaling deficits without obvious phenotypic abnormality attributable to Ipa(2). Molecular cellular biology. 2002;22(19):6921-9.

16. An S, Bleu T, Hallmark OG, Goetzl EJ. Characterization of a novel subtype of human G proteincoupled receptor for lysophosphatidic acid. J Biol Chem. 1998;273(14):7906-10.

17. Lee CW, Rivera R, Gardell S, Dubin AE, Chun J. GPR92 as a new G12/13- and Gq-coupled lysophosphatidic acid receptor that increases cAMP, LPA5. J Biol Chem. 2006;281(33):23589-97.

18. Fukushima K, Takahashi K, Kurokawa A, Ishimoto K, Otagaki S, Minami K, et al. Involvement of LPA receptor- 5 in the enhancement of cell motile activity by phorbol ester and anticancer drug treatments in melanoma A375cells. Biochem Biophys Res Commun. 2018;496(1):225-30.

19. Tang J, Kong D, Cui Q, Wang K, Zhang D, Yuan Q, et al. Bioinformatic analysis and identification of potential prognostic microRNAs and mRNAs in thyroid cancer. PeerJ. 2018;6:e4674. 
20. Liu L, He C, Zhou Q, Wang G, Lv Z, Liu J. Identification of key genes pathways of thyroid cancer by integrated bioinformatics analysis. 2019;234(12):23647-57.

21. Zhang S, Wang Q, Han Q, Han H, Lu P. Identification and analysis of genes associated with papillary thyroid carcinoma by bioinformatics methods. Bioscience reports. 2019;39(4).

22. Stavik B, Skretting G, Aasheim HC, Tinholt M, Zernichow L, Sletten M, et al. Downregulation of TFPI in breast cancer cells induces tyrosine phosphorylation signaling and increases metastatic growth by stimulating cell motility. BMC Cancer. 2011;11:357.

23. Stavik B, Tinholt M, Sletten M, Skretting G, Sandset PM, Iversen N. TFPlalpha and TFPIbeta are expressed at the surface of breast cancer cells and inhibit TF-FVIla activity. J Hematol Oncol. 2013;6:5.

24. Ravindranath TM, Goto M, lqbal O, Florian-Kujawski M, Hoppensteadt D, Hammadeh R, et al. Plasma thrombin activatable fibrinolysis inhibitor and tissue factor pathway inhibitor changes following sepsis. Clinical applied thrombosis/hemostasis: official journal of the International Academy of Clinical Applied Thrombosis/Hemostasis. 2007;13(4):362-8.

25. Wang T, Gilkes DM, Takano N, Xiang L, Luo W, Bishop CJ, et al. Hypoxia-inducible factors and RAB22A mediate formation of microvesicles that stimulate breast cancer invasion and metastasis. Proc Natl Acad Sci USA. 2014;111(31):E3234-42.

26. Cai B, Chen W, Pan Y, Chen H, Zhang Y, Weng Z, et al. Inhibition of microRNA-500 has anti-cancer effect through its conditional downstream target of TFPI in human prostate cancer. 2017;77(10):1057-65.

27. Tinholt M, Vollan HK, Sahlberg KK, Jernstrom S, Kaveh F, Lingjaerde OC, et al. Tumor expression, plasma levels and genetic polymorphisms of the coagulation inhibitor TFPI are associated with clinicopathological parameters and survival in breast cancer, in contrast to the coagulation initiator TF. Breast cancer research: BCR. 2015;17:44.

28. Zarychta E, Rhone P, Bielawski K, Rosc D, Szot K, Zdunska M, et al. Elevated plasma levels of tissue factor as a valuable diagnostic biomarker with relevant efficacy for prediction of breast cancer morbidity. Journal of physiology and pharmacology: an official journal of the Polish Physiological Society. 2018;69(6).

29. Erem C, Ucuncu O, Yilmaz M, Kocak M, Nuhoglu I, Ersoz HO. Increased thrombin-activatable fibrinolysis inhibitor and decreased tissue factor pathway inhibitor in patients with hypothyroidism. Endocrine. 2009;35(1):75-80.

30. Allard B, Longhi MS, Robson SC, Stagg J. The ectonucleotidases CD39 and CD73: Novel checkpoint inhibitor targets. Immunological reviews. 2017;276(1):121-44.

31. Mosaad Zaki E, Mohamed Zahran A, Abdelazeem Metwaly A, Hafez R, Hussein S, Elaiw Mohammed A. Impact of CD39 expression on CD4 + T lymphocytes and $6 q$ deletion on outcome of patients with chronic lymphocytic leukemia. Hematol Oncol Stem Cell Ther. 2019;12(1):26-31.

32. Cai XY, Wang XF, Li J, Dong JN, Liu JQ, Li NP, et al. High expression of CD39 in gastric cancer reduces patient outcome following radical resection. Oncology letters. 2016;12(5):4080-6. 
33. Mandapathil M, Boduc M, Roessler M, Guldner C, Walliczek-Dworschak U, Mandic R. Ectonucleotidase CD39 expression in regional metastases in head and neck cancer. Acta otolaryngologica. 2018;138(4):428-32.

34. Sun X, Han L, Seth P, Bian S, Li L, Csizmadia E, et al. Disordered purinergic signaling and abnormal cellular metabolism are associated with development of liver cancer in Cd39/ENTPD1 null mice. Hepatology. 2013;57(1):205-16.

\section{Figures}

A

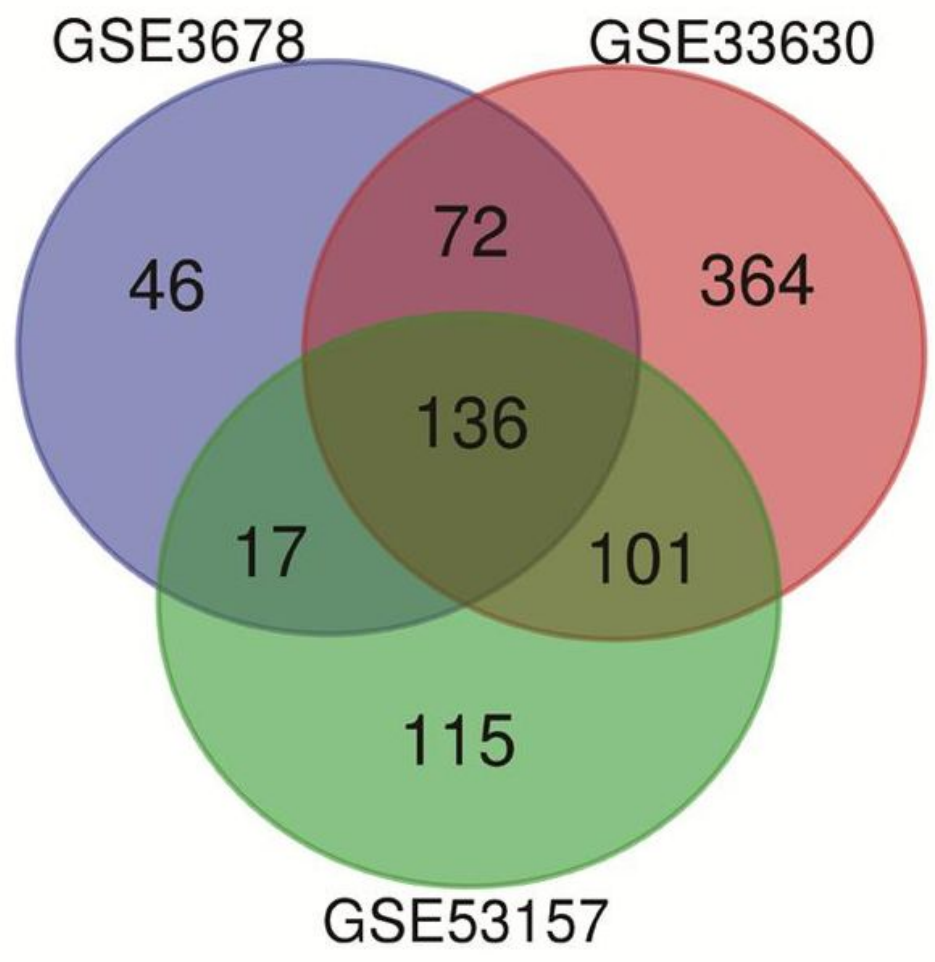

B

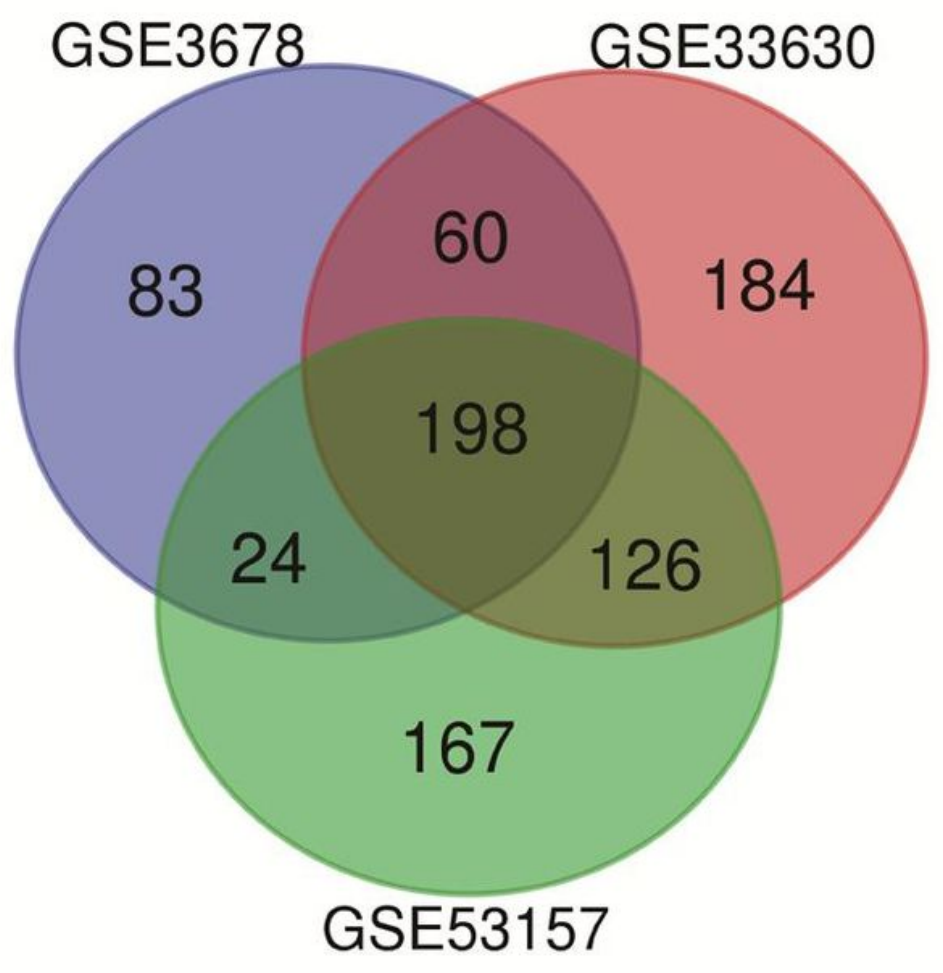

Figure 1

Selection of 334 common DEGs from the three datasets (GSE3678, GSE33630 and GSE53157). A. 136 DEGs were up-regulated (logFC囚1); B. 198 DEGs were down-regulated (logFC冈1) 
A

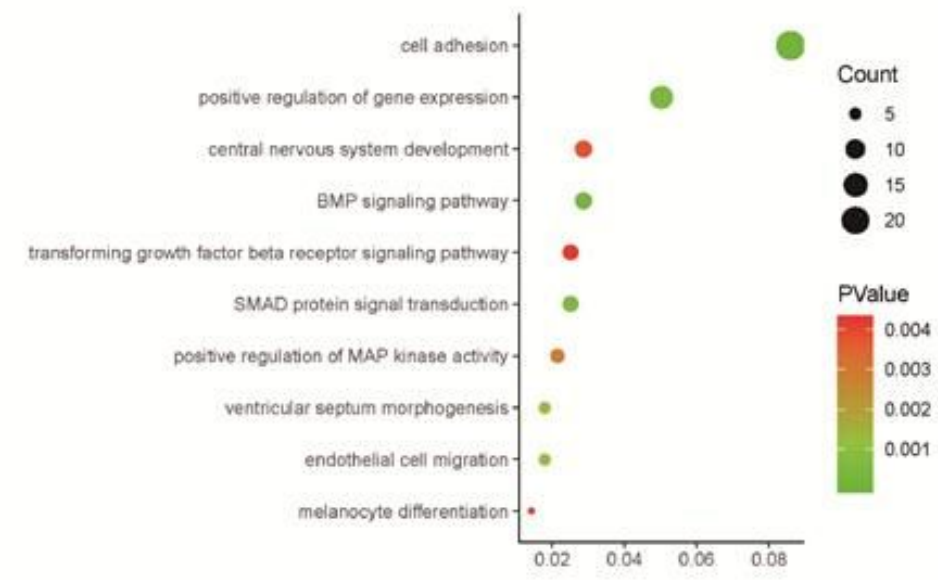

C

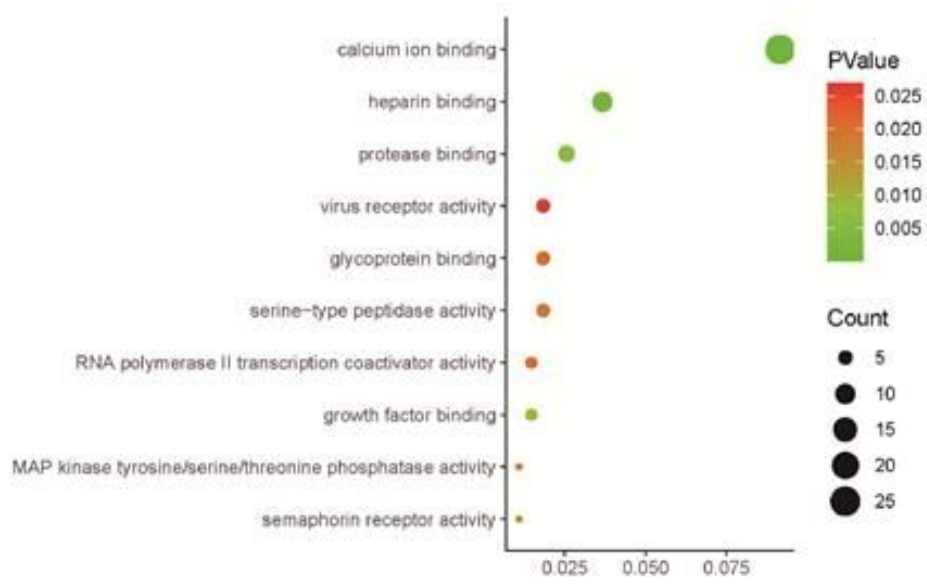

B

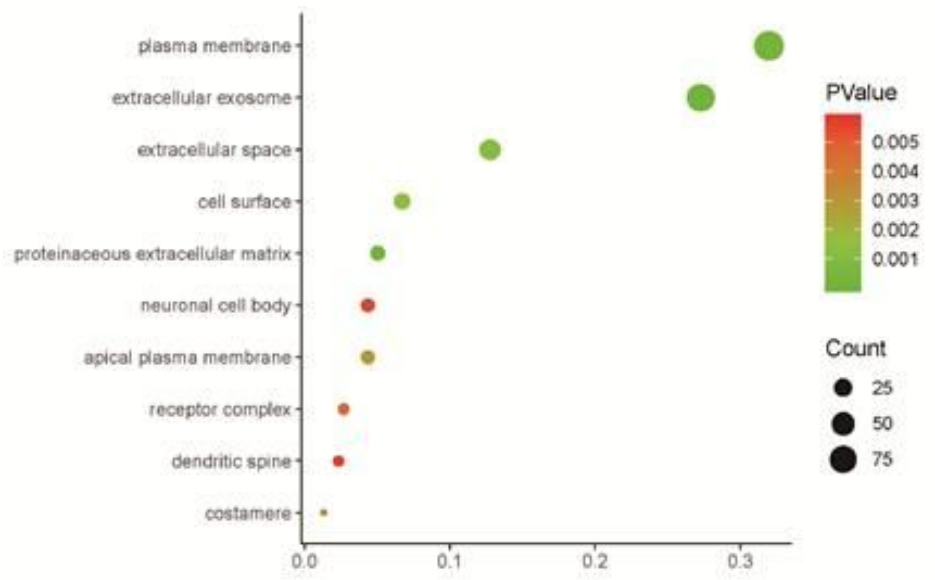

D

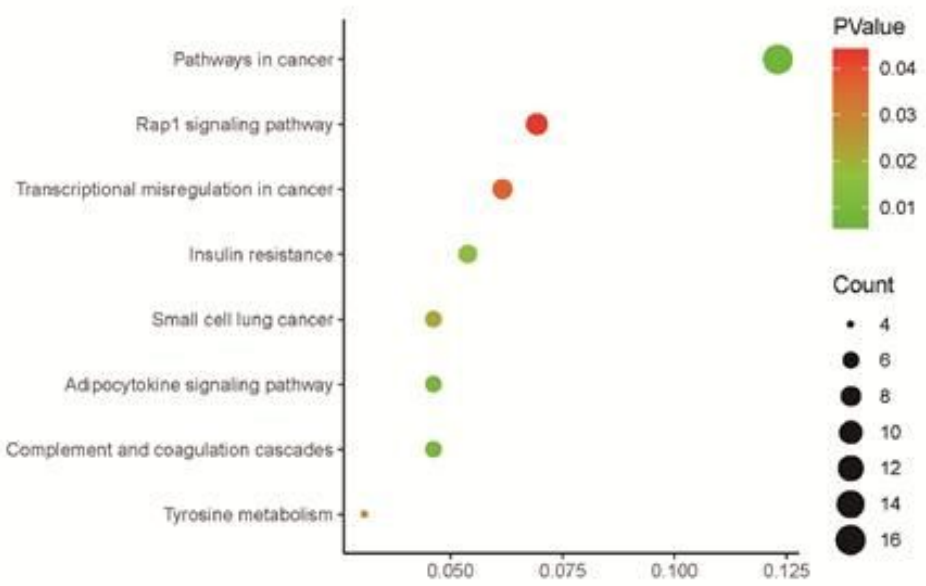

\section{Figure 2}

The top 10 gene ontology (GO) and significantly enriched KEGG pathways. A. BP; B. CC; C. MF; D. KEGG pathways. 


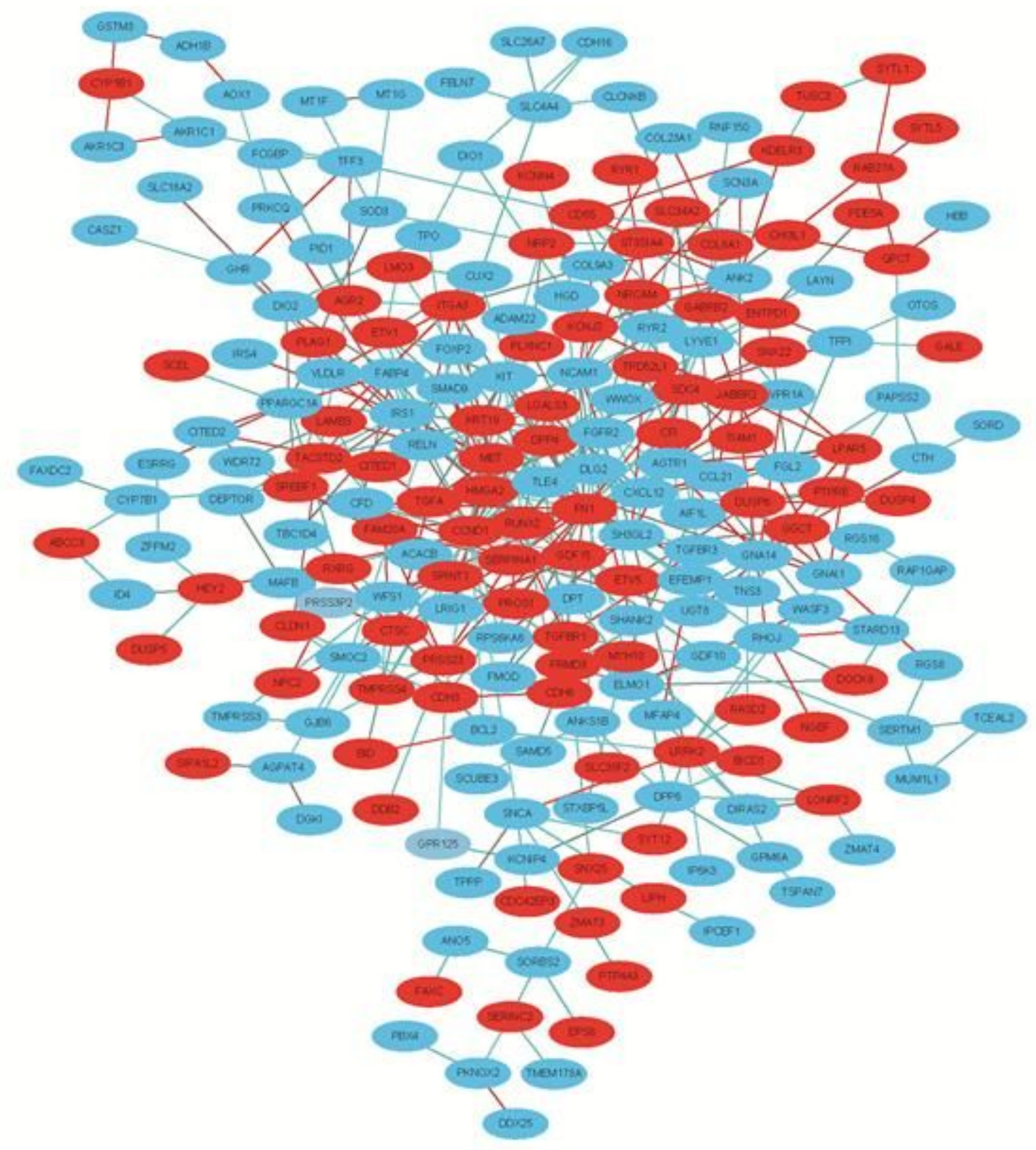

Figure 3

PPI network built by STRING and Cytoscape. Each nodel meant a protein module; the edges represented proteins interaction; red circles denoted up-regulated DEGs and blue circles represented down-regulated DEGs. 
A

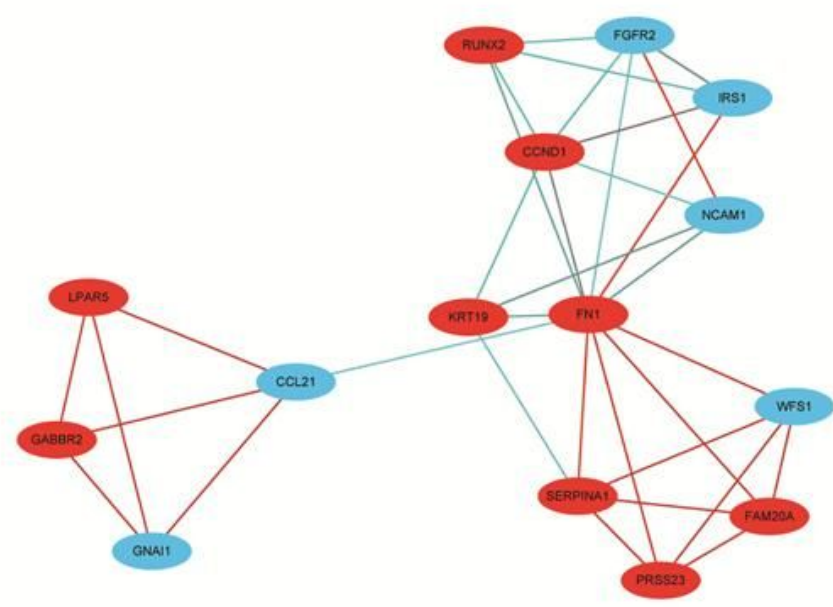

B

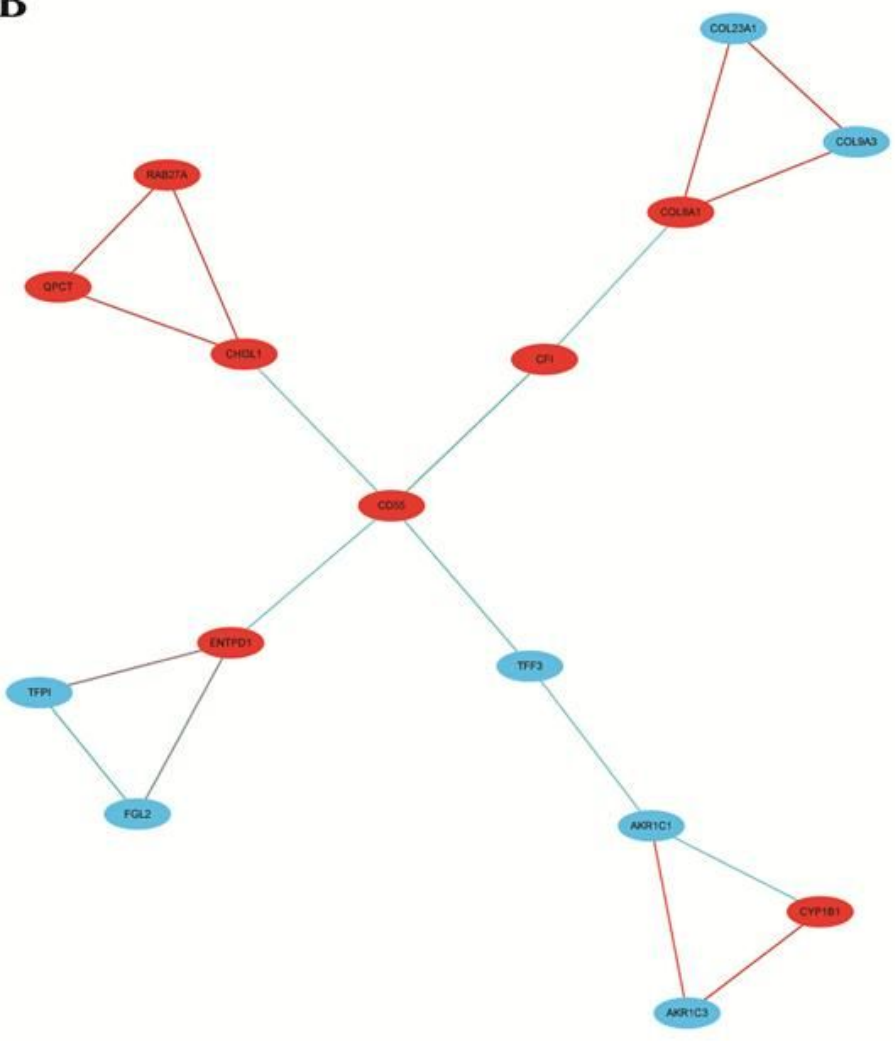

Figure 4

Module analysis by MCODE in the Cytoscape software. A. module1; B. module2. (red circles signified upregulated DEGs and blue circles denoted down-regulated DEGs) 
AKR1C3

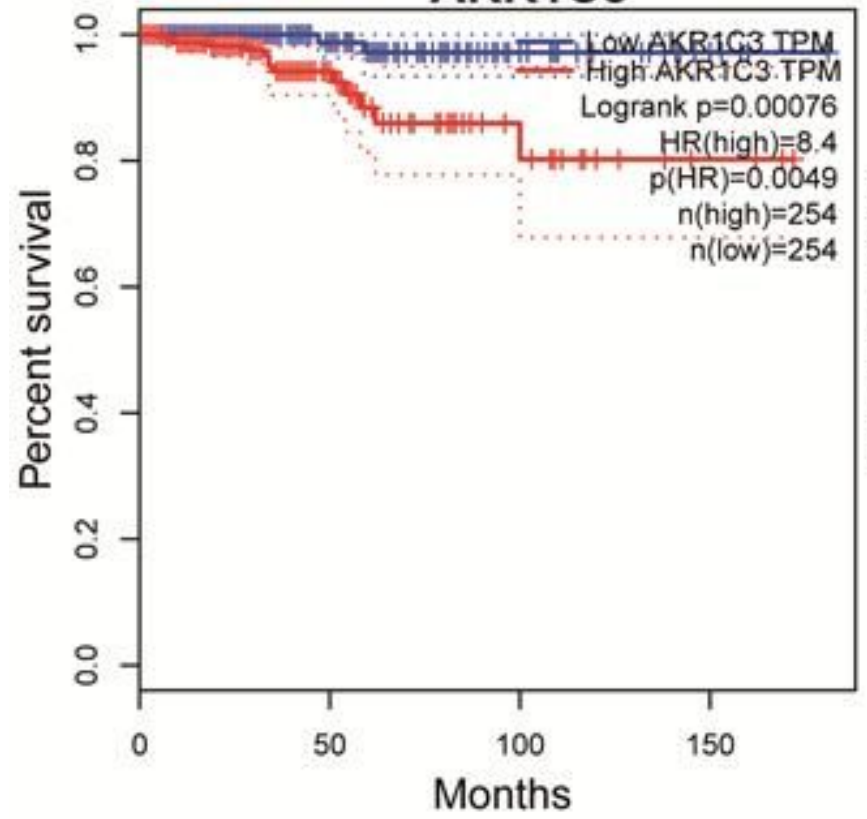

LPAR5

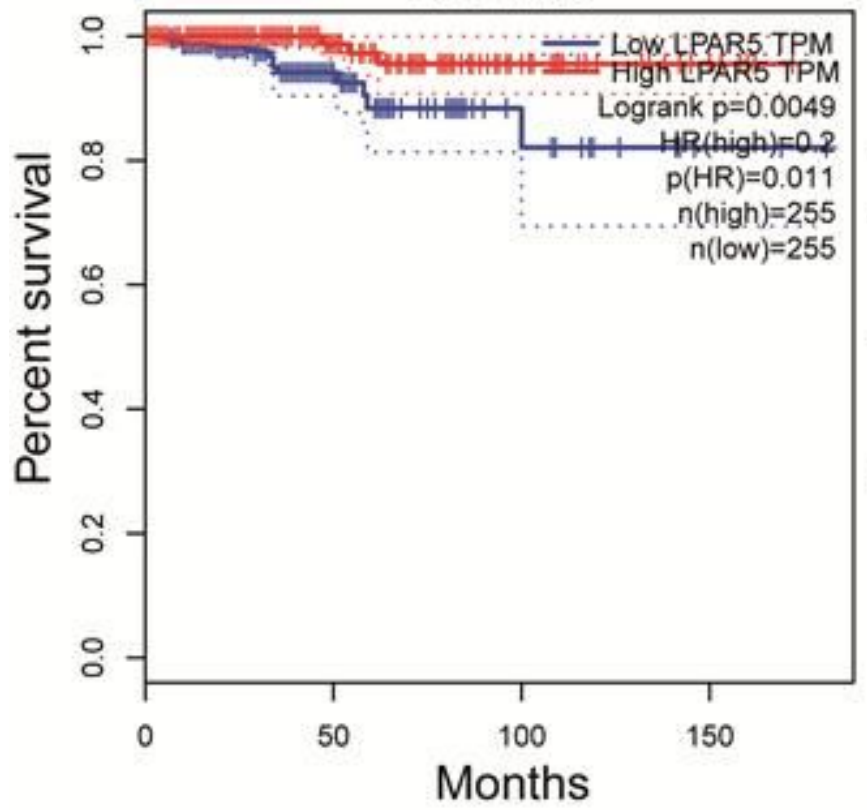

ENTPD1

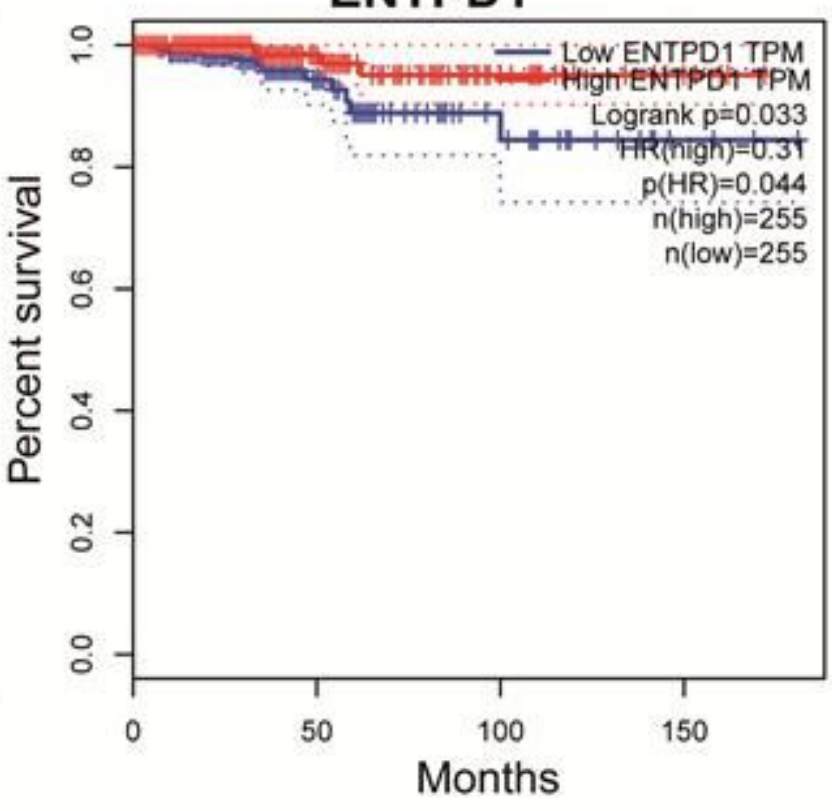

TFPI

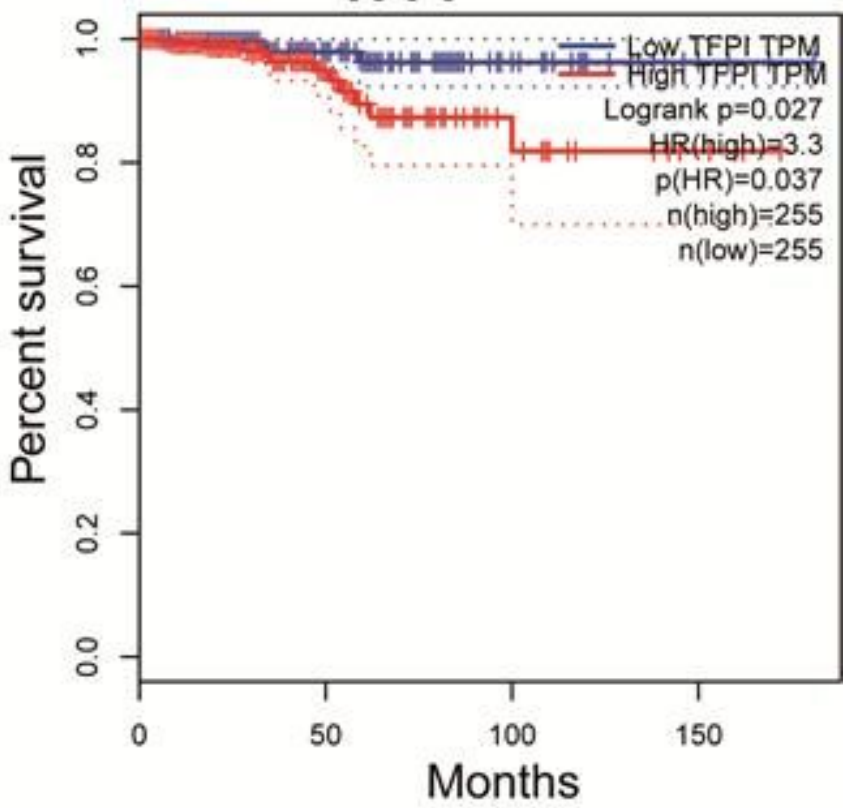

\section{Figure 5}

The association of the 4 hub genes expression with overall survival of patients with PTC via GEPIA analysis. 

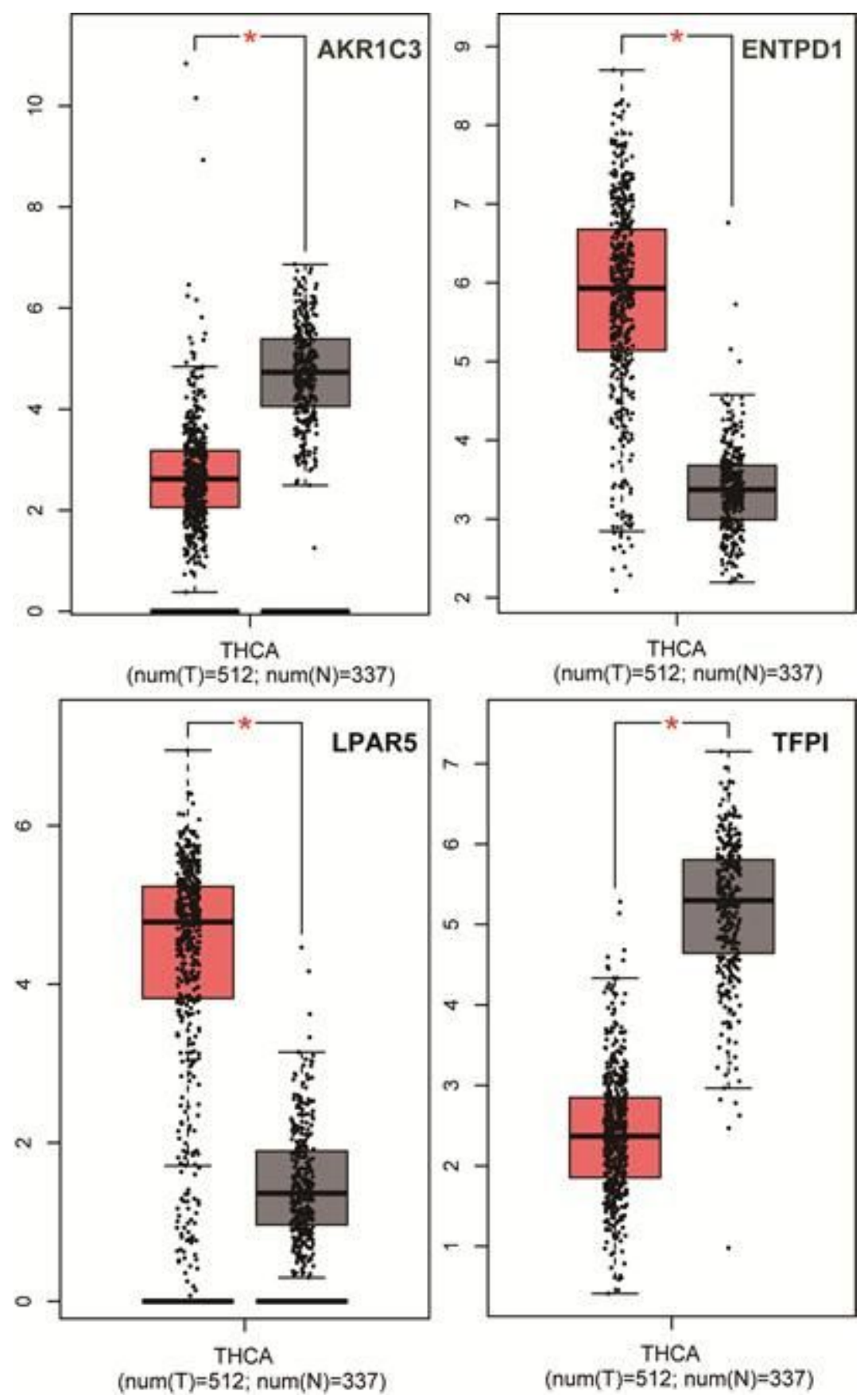

Figure 6

Analysis of hub genes expression levels with GEPIA analysis. 

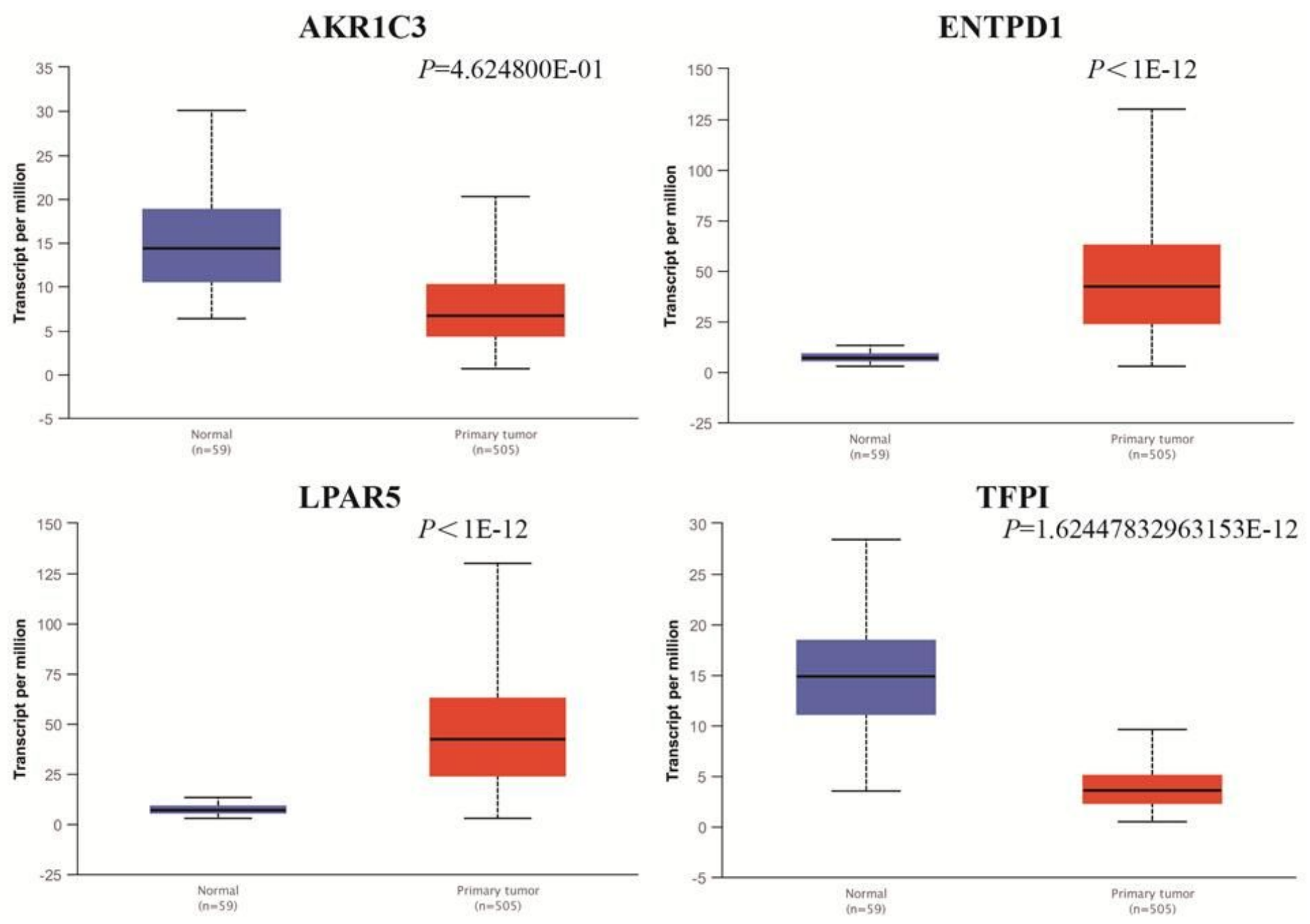

Figure 7

Analysis of hub genes expression levels with UALCAN analysis.

\section{Para-cancerous}

\section{Cancer}

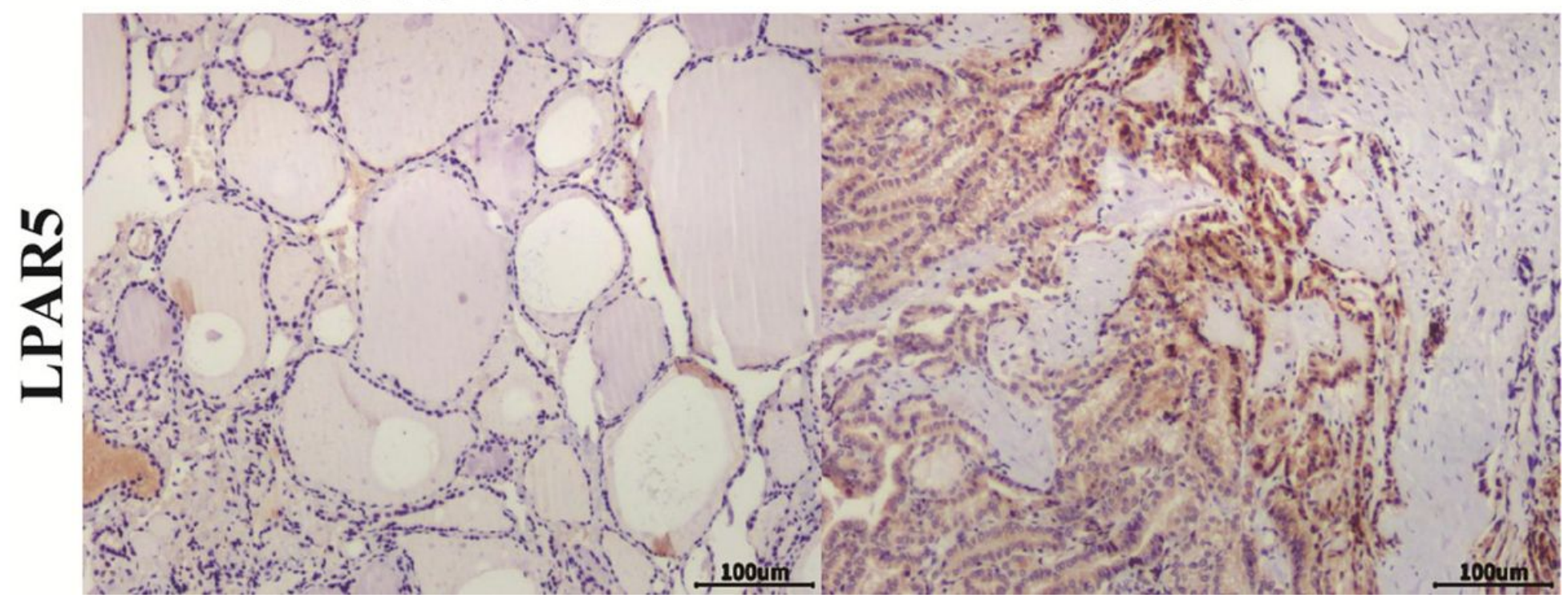

Figure 8 
Representative IHC for LPAR5 expression in tissue microarrays. (Scale bar, 100Пm) 